\title{
Upper bound on the cutoff in the Standard Model
}

M.A.Zubkov*

ITEP, B.Cheremushkinskaya 25, Moscow, 117259, Russia

E-mail: zubkoveitep.ru

\section{A.I.Veselov}

ITEP, B.Cheremushkinskaya 25, Moscow, 117259, Russia

E-mail: veseloveitep.ru

The main objective of this presentation is to point out that the Upper bound on the cutoff in lattice Electroweak theory is still unknown. The consideration of the continuum theory is based on the perturbation expansion around trivial vacuum. The internal structure of the lattice Weinberg Salam model may appear to be more complicated especially in the region of the phase diagram close to the phase transition between the physical Higgs phase and the unphysical symmetric phase of the lattice model, where the continuum physics is to be approached. We represent the results of our numerical investigation of the quenched model at infinite bare scalar self coupling $\lambda$. These results demonstrate that at $\lambda=\infty$ the upper bound on the cutoff is around $\frac{\pi}{a}=1.4$ Tev. The preliminary results for finite $\lambda$ are also presented. Basing on these results we cannot yet make a definite conclusion on the maximal value of the cutoff admitted in the lattice model, although we have found that the cutoff cannot exceed the value around $1.4 \pm 0.2 \mathrm{Tev}$ for a certain particular choice of the couplings $\left(\lambda=0.009, \beta=12, \theta_{W}=30^{\circ}\right)$ for the lattices of sizes up to $12^{3} \times 16$. We also observe that the topological defects, which are to be identified with quantum Nambu monopoles, dominate in vacuum in the vicinity of the transition. This indicates that the vacuum of the model is different from the trivial one. In addition we remind the results of the previous numerical investigations of the $S U(2)$ gauge - Higgs model, where the maximal reported value of the cutoff was around $1.5 \mathrm{Tev}$.

The XXVII International Symposium on Lattice Field Theory - LAT2009

July 26-31 2009

Peking University, Beijing, China

\footnotetext{
${ }^{*}$ Speaker.
} 


\section{Introduction}

According to the conventional point of view the upper bound $\Lambda$ on the cutoff in the Electroweak theory (without fermions) depends on the Higgs mass. It is decreased when the Higgs mass is increased. And at the Higgs mass around $1 \mathrm{Tev} \Lambda$ becomes of the order of $M_{H}$. At the same time for $M_{H} \sim 200 \mathrm{Gev}$ the value of $\Lambda$ can be made almost infinite ${ }^{1}$. This conclusion is made basing on the perturbation expansion around trivial vacuum. In our presentation we demonstrate that the vacuum of the lattice Weinberg - Salam model is rather complicated, which means that the application of the perturbation expansion around trivial vacuum may be limited.

Namely, we investigate the behavior of the topological defects composed of the lattice gauge fields that are to be identified with quantum Nambu monopoles $[1,2,3]$. We show that their lattice density increases along the lines of constant physics when the ultraviolet cutoff in increased. At sufficiently large values of the cutoff these objects begin to dominate.

Moving further along the line of constant physics we reach the point on the phase diagram where the monopole worldlines begin to percolate. This point roughly coincides with the position of the transition between the physical Higgs phase and the unphysical symmetric phase of the lattice model. At infinite bare scalar self coupling $\lambda$ the transition is a crossover and the ultraviolet cutoff achieves its maximal value around $1.4 \mathrm{Tev}$ at the transition point. At smaller bare values of $\lambda$ correspondent to small Higgs masses the phase transition becomes stronger. Still we do not know the order of the phase transition at small values of $\lambda$. We have estimated the maximal value of the cutoff in the vicinity of the transition point at $\lambda=0.009$. The obtained value of the cutoff appears to be around $1.4 \mathrm{Tev}$.

\section{The lattice model under investigation}

The lattice Weinberg - Salam Model without fermions contains gauge field $\mathscr{U}=(U, \theta)$ (where $U \in S U(2), \quad e^{i \theta} \in U(1)$ are realized as link variables), and the scalar doublet $\Phi_{\alpha},(\alpha=1,2)$ defined on sites.

The action is taken in the form

$$
\begin{aligned}
S= & \beta \sum_{\text {plaquettes }}\left(\left(1-\frac{1}{2} \operatorname{Tr} U_{p}\right)+\frac{1}{\operatorname{tg}^{2} \theta_{W}}\left(1-\cos \theta_{p}\right)\right)+ \\
& -\gamma \sum_{x y} \operatorname{Re}\left(\Phi^{+} U_{x y} e^{i \theta_{x y}} \Phi\right)+\sum_{x}\left(\left|\Phi_{x}\right|^{2}+\lambda\left(\left|\Phi_{x}\right|^{2}-1\right)^{2}\right),
\end{aligned}
$$

where the plaquette variables are defined as $U_{p}=U_{x y} U_{y z} U_{w z}^{*} U_{x w}^{*}$, and $\theta_{p}=\theta_{x y}+\theta_{y z}-\theta_{w z}-\theta_{x w}$ for the plaquette composed of the vertices $x, y, z, w$. Here $\lambda$ is the scalar self coupling, and $\gamma=2 \kappa$, where $\kappa$ corresponds to the constant used in the investigations of the $S U(2)$ gauge Higgs model. $\theta_{W}$ is the Weinberg angle. Bare fine structure constant $\alpha$ is expressed through $\beta$ and $\theta_{W}$ as $\alpha=$ $\frac{\operatorname{tg}^{2} \theta_{W}}{\pi \beta\left(1+\operatorname{tg}^{2} \theta_{W}\right)}$. In our investigation we fix bare Weinberg angle equal to $30^{\circ}$. The renormalized fine structure constant can be extracted through the potential for the infinitely heavy external charged particles.

\footnotetext{
${ }^{1}$ Here we do not consider vacuum stability bound on the Higgs mass related to the fermion loops.
} 


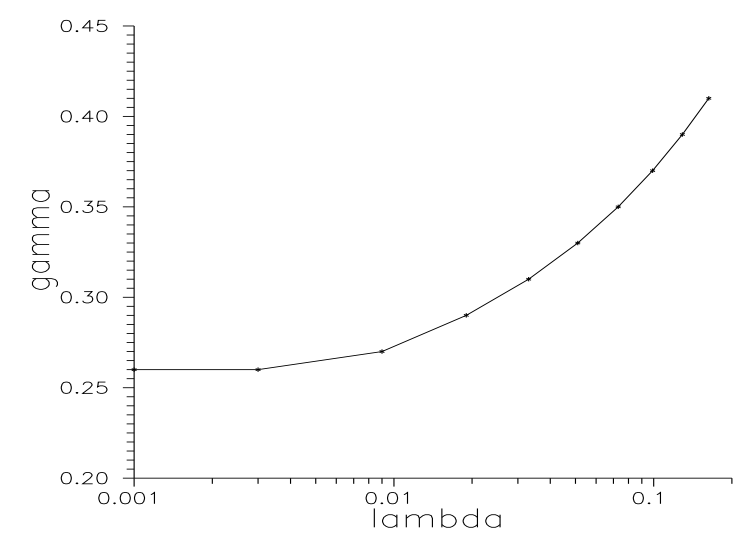

Figure 1: The phase diagram of the model in the $(\gamma, \lambda)$-plane at $\beta=12$.

\section{Phase diagram}

The phase diagram at infinite $\lambda$ is represented in [4]. Qualitatively the phase diagram at finite $\lambda$ looks similar to that of infinite $\lambda$. In the three - dimensional $(\beta, \gamma, \lambda)$ phase diagram the transition surfaces are two - dimensional. The lines of constant physics on the tree level are the lines $\left(\frac{\lambda}{\gamma^{2}}=\frac{1}{8 \beta} \frac{M_{H}^{2}}{M_{W}^{2}}=\right.$ const; $\beta=\frac{1}{4 \pi \alpha}=$ const). In general the cutoff is increased along the line of constant physics when $\gamma$ is decreased. The maximal value of the cutoff is achieved at the transition point. Nambu monopole density in lattice units is also increased when the ultraviolet cutoff is increased.

At $\beta=12$ the phase diagram is represented on Fig. 2. The physical Higgs phase is situated up to the transition line. The position of the transition is localized at the point where the susceptibility extracted from the Higgs field creation operator achieves its maximum.

All simulations were performed on lattices of sizes $8^{3} \times 16$. Several points were checked using larger lattices up to $16^{3} \times 24$. At $\lambda=\infty$ we found no significant difference between the results obtained using the mentioned lattices. For small $\lambda$ the careful investigation of the dependence of physical observables on the lattice size has not been performed.

\section{Calculation of the cutoff}

The following variable is considered as creating the $Z$ boson: $Z_{x y}=Z_{x}^{\mu}=\sin \left[\operatorname{Arg}\left(\Phi_{x}^{+} U_{x y} e^{i \theta_{x y}} \Phi_{y}\right)\right]$. In order to evaluate the masses of the $Z$-boson and the Higgs boson we use the correlators:

$$
\frac{1}{N^{6}} \sum_{\bar{x}, \bar{y}}\left\langle\sum_{\mu} Z_{x}^{\mu} Z_{y}^{\mu}\right\rangle \sim e^{-M_{Z}\left|x_{0}-y_{0}\right|}+e^{-M_{Z}\left(L-\left|x_{0}-y_{0}\right|\right)}
$$

and

$$
\frac{1}{N^{6}} \sum_{\bar{x}, \bar{y}}\left(\left\langle H_{x} H_{y}\right\rangle-\langle H\rangle^{2}\right) \sim e^{-M_{H}\left|x_{0}-y_{0}\right|}+e^{-M_{H}\left(L-\left|x_{0}-y_{0}\right|\right)}
$$


Here the summation $\sum_{\bar{x}, \bar{y}}$ is over the three "space" components of the four - vectors $x$ and $y$ while $x_{0}, y_{0}$ denote their "time" components. $N$ is the lattice length in "space" direction. $L$ is the lattice length in the "time" direction.

In lattice calculations we used two different operators that create Higgs bosons: $H_{x}=|\Phi|$ and $H_{x}=\sum_{y} Z_{x y}^{2}$. In both cases $H_{x}$ is defined at the site $x$, the sum $\sum_{y}$ is over its neighboring sites $y$.

After fixing the unitary gauge, lattice Electroweak theory becomes a lattice $U(1)$ gauge theory. The $U(1)$ gauge field is $A_{x y}=A_{x}^{\mu}=\left[-\operatorname{Arg}\left(\Phi_{x}^{+} U_{x y} e^{i \theta_{x y}} \Phi_{y}\right)+2 \theta_{x y}\right] \bmod 2 \pi$. The usual Electromagnetic field is $A_{\mathrm{EM}}=A+Z^{\prime}-2 \sin ^{2} \theta_{W} Z^{\prime}$, where $Z^{\prime}=\left[\operatorname{Arg}\left(\Phi_{x}^{+} U_{x y} e^{i \theta_{x y}} \Phi_{y}\right)\right] \bmod 2 \pi$.

The physical scale is given in our lattice theory by the value of the $Z$-boson mass $M_{Z}^{\text {phys }} \sim 91$ $\mathrm{GeV}$. Therefore the lattice spacing is evaluated to be $a \sim[91 \mathrm{GeV}]^{-1} M_{Z}$, where $M_{Z}$ is the $Z$ boson mass in lattice units.

At infinite $\lambda$ the real continuum physics should be approached along the the line of constant $\alpha_{R}=\frac{1}{128}$. The ultraviolet cutoff is $\Lambda=\frac{\pi}{a}=(\pi \times 91 \mathrm{GeV}) / M_{Z} . \Lambda$ is increased slowly along this line with decreasing $\gamma$ and achieves the value around $1.35 \mathrm{TeV}$ at the transition point between the physical Higgs phase and the symmetric phase. According to our results this value does not depend on the lattice size.

In the region of the phase diagram represented on Fig. 2 the situation is similar. Our data obtained on the lattice $8^{3} \times 16$ shows that $\Lambda$ is increased slowly with the decrease of $\gamma$ at any fixed $\lambda$. We investigated carefully the vicinity of the transition point at fixed $\lambda=0.009$ and $\beta=12$. It has been found that at the transition point the value of $\Lambda$ is equal to $1.4 \pm 0.2 \mathrm{Tev}$. The first check of a larger lattice (of size $12^{3} \times 16$ ) does not show an increase of this value. However, the careful investigation of the dependence of $\Lambda$ on the lattice size (as well as on $\lambda$ ) is to be the subject of future investigations.

On Fig. 3 the dependence of $M_{Z}$ in lattice units on $\gamma$ is represented at $\lambda=0.009$ and $\beta=12$, where $\gamma_{c}=0.273 \pm 0.002$. . Unfortunately, we cannot yet estimate the renormalized Higgs boson mass due to the lack of statistics. However, we expect it does not deviate significantly from the tree level estimate $M_{H}^{0}=\frac{\sqrt{8 \beta \lambda}}{\gamma} \times 80 \mathrm{Gev}$. In the vicinity of the phase transition at $\lambda=0.009, \beta=12$ bare value of the Higgs mass is $M_{H}^{0} \sim 270 \mathrm{Gev}$.

\section{The renormalized coupling}

The bare constant $\alpha=e^{2} / 4 \pi$ (where $e$ is the electric charge) can be easily calculated in our lattice model. It is found to be equal to $1 /(4 \pi \beta)$. Therefore, its physical value $\alpha\left(M_{Z}\right) \sim 1 / 128$ could be achieved at the values of $\beta$ in some vicinity of 10 . This naive guess is, however, to be corrected by the calculation of the renormalized coupling constant $\alpha_{R}$. We perform this calculation using the potential for infinitely heavy external fermions. We consider Wilson loops for the righthanded external leptons: $\mathscr{W}_{\text {lept }}^{\mathrm{R}}(l)=\left\langle\operatorname{Re} \Pi_{(x y) \in l} e^{2 i \theta_{x y}}\right\rangle$. Here $l$ denotes a closed contour on the lattice. We consider the following quantity constructed from the rectangular Wilson loop of size $r \times t$ : $\mathscr{V}(r)=\lim _{t \rightarrow \infty} \log _{\mathscr{W}(r \times(t+1))}$. At large enough distances we expect the appearance of the Coulomb interaction $\mathscr{V}(r)=-\frac{\alpha_{R}}{r}+$ const.

The renormalized coupling constant $\alpha$ is found to be close to the realistic value $\alpha\left(M_{Z}\right)=$ $1 / 128$ along the line represented in Fig. 1 (at $\lambda \rightarrow \infty$ ) in the vicinity of $\beta=15$. We do not observe any dependence of $\alpha_{R}$ on the lattice size at $\lambda=\infty$. 


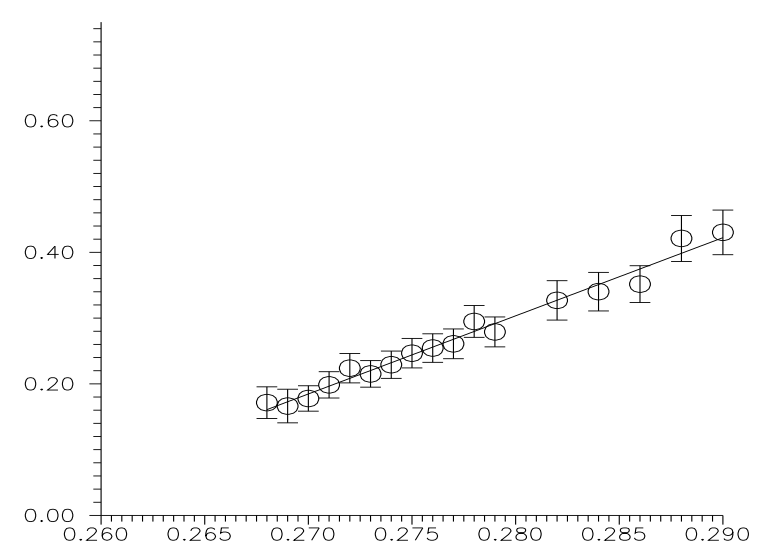

Figure 2: $\mathrm{Z}$ - boson mass in lattice units at $\lambda=0.009$ and $\beta=12$.

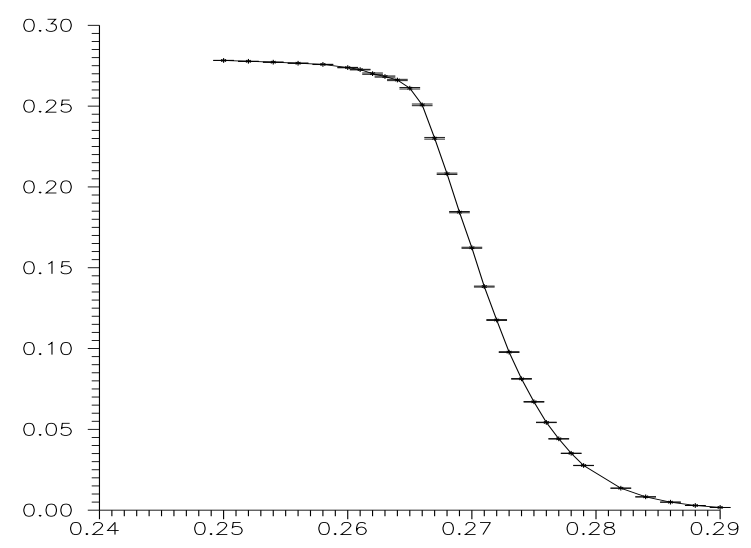

Figure 3: Nambu monopole density as a function of $\gamma$ at $\lambda=0.009, \beta=12$.

At $\lambda=0.009, \beta=12, \gamma=\gamma_{c}(\lambda) \sim 0.273$ the renormalized fine structure constant calculated on the lattice $8^{3} \times 16$ is $\alpha_{R}=\frac{1}{98 \pm 3}$. The same value has been obtained also on the larger lattice $\left(12^{3} \times 16\right)$, which shows that the value of $\alpha_{R}$ does not depend on the lattice size also for the small values of $\lambda$.

\section{Nambu monopole density}

According to $[2,3,4]$ the worldlines of the quantum Nambu monopoles can be extracted from the field configurations as follows:

$$
j_{A}=\frac{1}{2 \pi} * d([d A] \bmod 2 \pi)
$$

(The notations of differential forms on the lattice [5] are used here.) The monopole density is defined as $\rho=\left\langle\frac{\sum_{\text {links }}\left|j_{\text {link }}\right|}{4 L^{4}}\right\rangle$, where $L$ is the lattice size. 


\begin{tabular}{|c|c|c|}
\hline Reference & inverse lattice spacing $\frac{1}{a}(\mathrm{GeV})$ & $M_{H}(\mathrm{GeV})$ \\
\hline$[6]$ & 140 (space direction) 570 (time direction) & 80 \\
\hline$[7]$ & 280 (time direction) & 80 \\
\hline$[8]$ & 280 & 34 \\
\hline$[9]$ & 110 & 16 \\
\hline$[10]$ & 90 (space direction) 350 (time direction) & 34 \\
\hline$[11]$ & 280 & 48 \\
\hline$[12]$ & 140 & 35 \\
\hline$[13]$ & 280 & 20,50 \\
\hline$[14]$ & 190 & 50 \\
\hline$[15]$ & 260 & $57-85$ \\
\hline$[16]$ & $200-300$ & $47-108$ \\
\hline$[17]$ & 400 & 480 \\
\hline$[18]$ & $330-470$ & $280-720$ \\
\hline$[19]$ & $250-470$ & $720(\lambda=\infty)$ \\
\hline
\end{tabular}

In Fig. 4 we represent Nambu monopole density as a function of $\gamma$ at $\lambda=0.009, \beta=12$. The point of the transition is localized as the position of the maximum of the susceptibility $\chi=$ $\left\langle H^{2}\right\rangle-\langle H\rangle^{2}$ extracted from $H=\sum_{y} Z_{x y}^{2}$. The value of monopole density at $\gamma_{c}=0.273, \beta=12$, $\lambda=0.009$ is around 0.1. At this point the value of the cutoff is $\Lambda \sim 1.4 \pm 0.2 \mathrm{Tev}$. The monopole density around 0.1 means that among 10 sites there exist 4 sites that are occupied by the monopole. Average distance between the two monopoles is, therefore, less than 1 lattice spacing and it is not possible at all to speak of the given configurations as of representing the single Nambu monopole.

\section{Conclusions}

In Table 1 we list the values of the lattice spacing used in selected lattice studies of $S U(2)$ Gauge - Higgs Model. From this table it is clear that the correspondent value of the cutoff $\frac{\pi}{a}$ does not exceed 1.5 Tev.

Our own numerical data demonstrate that the vacuum structure of the lattice Weinberg - Salam model is rather complicated. Namely, the topological defects identified with quantum Nambu monopoles dominate in vacuum in the vicinity of the phase transition between the symmetric phase and the Higgs phase. This indicates that the usual perturbation expansion around trivial vacuum may not be applied in this region of the phase diagram. As a consequence one cannot apply the conventional perturbation theory to the evaluation of the Ultraviolet cutoff upper bound in this region of the phase diagram. Qualitatively this situation seem to us similar to that of the Ginzburg Landau theory of superconductivity. Within this theory in a certain vicinity of the phase transition the fluctuations of the order parameter become so strong that the perturbation expansion around the trivial solution of Ginzburg - Landau equations cannot be applied.

Thus we conclude that the upper bound on the Ultraviolet cutoff in the lattice Electroweak theory is still not known. The establishing of this upper bound is to be a subject of future investiga- 
tions. We suppose that the upper bound on the cutoff obtained with the aid of nonperturbative lattice methods may differ from the conventional one obtained via the perturbation expansion around trivial vacuum.

\section{Acknowledgments}

This work was partly supported by RFBR grants 09-02-08308, 09-02-00338, 08-02-00661, and 07-02-00237, by Grant for leading scientific schools 679.2008.2, by Federal Program of the Russian Ministry of Industry, Science and Technology No 40.052.1.1.1112. The numerical simulations have been performed using the facilities of Moscow Joint Supercomputer Center.

\section{References}

[1] Y. Nambu, Nucl.Phys. B 130, 505 (1977);

Ana Achucarro and Tanmay Vachaspati, Phys. Rept. 327, 347 (2000); Phys. Rept. 327, 427 (2000).

[2] B.L.G. Bakker, A.I. Veselov, and M.A. Zubkov, Phys. Lett. B 583, 379 (2004); Yad. Fiz. 68, 1045 (2005); Phys. Lett. B 620, 156 (2005); Phys. Lett. B 642, 147 (2006); J. Phys. G: Nucl. Part. Phys. 36 (2009) 075008; arXiv:0708.2864, PoSLAT2007:337,2007

[3] M.N. Chernodub, JETP Lett. 66, 605 (1997)

[4] A.I. Veselov, and M.A. Zubkov, JHEP 0812:109 (2008);

[5] M.I. Polikarpov, U.J. Wiese, and M.A. Zubkov, Phys. Lett. B 309, 133 (1993).

[6] F. Csikor, Z. Fodor, J. Heitger Phys.Rev.Lett. 82 (1999) 21-24 Phys.Rev. D58 (1998) 094504 Nucl.Phys.Proc.Suppl. 63 (1998) 569-571

[7] F. Csikor, Z. Fodor, J. Heitger Phys.Lett. B441 (1998) 354-362

[8] F. Csikor, Z. Fodor, J. Hein, A. Jaster, I. Montvay Nucl.Phys. B474 (1996) 421-445

[9] Joachim Hein (DESY), Jochen Heitger, Phys.Lett. B385 (1996) 242-248

[10] F. Csikor, Z. Fodor, J. Hein, J. Heitger, A. Jaster, I. Montvay Nucl.Phys.Proc.Suppl. 53 (1997) 612-614

[11] Z. Fodor, J. Hein, K. Jansen, A. Jaster, I. Montvay Nucl.Phys. B439 (1995) 147-186

[12] F. Csikor, Z. Fodor, J. Hein, J. Heitger, Phys.Lett. B357 (1995) 156-162

[13] F. Csikor, Z. Fodor, J. Hein, K.Jansen, A. Jaster, I. Montvay Nucl.Phys.Proc.Suppl. 42 (1995) 569-574

[14] F. Csikor, Z. Fodor, J. Hein, K.Jansen, A. Jaster, I. Montvay Phys.Lett. B334 (1994) 405-411

[15] Y. Aoki, F. Csikor, Z. Fodor, A. Ukawa Phys.Rev. D60 (1999) 013001 Nucl.Phys.Proc.Suppl. 73 (1999) 656-658

[16] Y. Aoki Phys.Rev. D56 (1997) 3860-3865

[17] W.Langguth, I.Montvay, P.Weisz Nucl.Phys.B277:11,1986.

[18] W. Langguth, I. Montvay (DESY) Z.Phys.C36:725,1987

[19] Anna Hasenfratz, Thomas Neuhaus, Nucl.Phys.B297:205,1988 